\title{
Multifunctional Platinum@BSA-Rapamycin Nanocarriers for the Combinatorial Therapy of Cerebral Cavernous Malformation
}

\author{
Elisa De Luca, ${ }^{\dagger}$ Deborah Pedone, ${ }^{\dagger, \dagger}$ Mauro Moglianetti, ${ }^{\dagger}$ Daniele Pulcini, ${ }^{\dagger}$ Andrea Perrelli, ${ }^{\S}, \|$

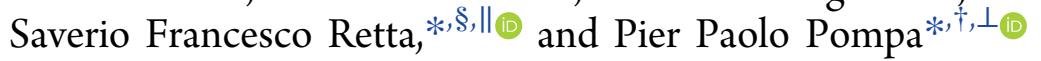 \\ ${ }^{\dagger}$ Nanobiointeractions \& Nanodiagnostics, Center for Biomolecular Nanotechnologies, Istituto Italiano di Tecnologia, Via Barsanti \\ 14, Arnesano Lecce 73010, Italy \\ ${ }^{\ddagger}$ Department of Engineering for Innovation, University of Salento, Via per Monteroni, Lecce 73100, Italy \\ ${ }^{\S}$ Department of Clinical and Biological Sciences, University of Torino, Regione Gonzole 10, Orbassano (Torino) 10043, Italy \\ "CCM Italia Research NetworkUniversity of Torino, Regione Gonzole 10, Orbassano (Torino) 10043, Italy \\ ${ }^{\perp}$ Nanobiointeractions \& Nanodiagnostics, Istituto Italiano di Tecnologia, Via Morego 30, Genova 16163, Italy
}

Supporting Information

\begin{abstract}
Platinum nanoparticles (PtNPs) are antioxidant enzymemimetic nanomaterials with significant potential for the treatment of complex diseases related to oxidative stress. Among such diseases, Cerebral Cavernous Malformation (CCM) is a major cerebrovascular disorder of genetic origin, which affects at least $0.5 \%$ of the general population. Accumulated evidence indicates that loss-of-function mutations of the three known CCM genes predispose endothelial cells to oxidative stress-mediated dysfunctions by affecting distinct redoxsensitive signaling pathways and mechanisms, including pro-oxidant and antioxidant pathways and autophagy. A multitargeted combinatorial therapy might thereby represent a promising strategy for the effective treatment of this disease. Herein, we developed a multifunctional nanocarrier by combining the radical scavenging activity of PtNPs with the autophagy-stimulating activity of rapamycin (Rapa). Our results show that the combinatorial targeting of redox signaling and autophagy dysfunctions is effective in rescuing major molecular and cellular hallmarks of CCM disease, suggesting its potential for the treatment of this and other oxidative stress-related diseases.

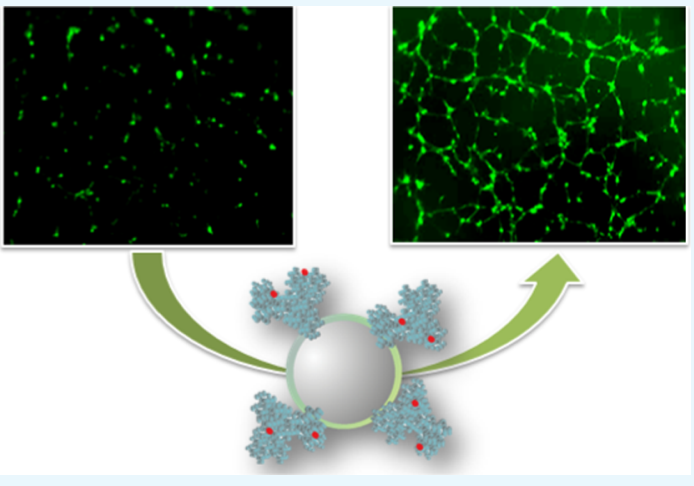

\section{INTRODUCTION}

Multifactorial diseases, such as cerebrovascular pathologies, require complex therapeutic approaches based on the combination of different drugs simultaneously directed at multiple targets to achieve synergy and efficient therapeutic response. ${ }^{1-4}$ Among cerebrovascular diseases, Cerebral Cavernous Malformation (CCM) has emerged as a major health issue, affecting approximately 24 million people worldwide. ${ }^{5-7}$ CCM is characterized by vascular lesions consisting of abnormally dilated and fragile blood capillaries, mainly occurring in the central nervous system, which expose to a lifetime risk of seizures, focal neurological deficits, and hemorrhagic stroke. ${ }^{5-8}$ Genetic studies have demonstrated that CCM is caused by loss-of-function mutation in three CCM genes (KRIT1/CCM1, CCM2, and CCM3 $)^{9}$ implicated in the maintenance of endothelial cell-cell junction stability and blood-brain barrier (BBB) integrity. ${ }^{10}$ Growing evidence suggests that multiple factors contribute to CCM disease pathogenesis and its highly variable expressivity. ${ }^{11}$ Indeed, it has been shown that loss-of-function of CCM genes affects major pleiotropic mechanisms involved in cellular redox homeostasis and defense against oxidative stress, including the fine-tuned crosstalk between redox signaling and autophagy. ${ }^{10}$ In particular, a recent report demonstrates that KRIT1 loss-of-function causes defective autophagy and consequent molecular and cellular abnormalities, including accumulation of the autophagy adapter p62, and abnormal persistence of dysfunctional mitochondria, leading to a strong increase in intracellular levels of reactive oxygen species (ROS). ${ }^{12-14}$ Importantly, these findings have opened novel perspectives for the pharmacological treatment of CCM disease, suggesting that a promising possibility would be the development of a combinatorial treatment that may simultaneously and synergistically target autophagic dysfunction and antioxidant deficiency. ${ }^{10,14}$ Consistently, all the potential therapeutic compounds for CCM disease proposed so far, including statins, ${ }^{15} \mathrm{~N}$-acetylcysteine, ${ }^{16,17}$ fasudil, ${ }^{18}$ tempol, ${ }^{19}$ vitamin D3, ${ }^{19}$ sulindac derivatives, ${ }^{20}$ rapamycin (Rapa), ${ }^{12}$ and avenanthramides, ${ }^{21,22}$ are endowed with either autophagyinducing or antioxidant properties or both. .0,13,14 $^{2}$

Received: July 13, 2018

Accepted: October 24, 2018

Published: November 13, 2018 
A

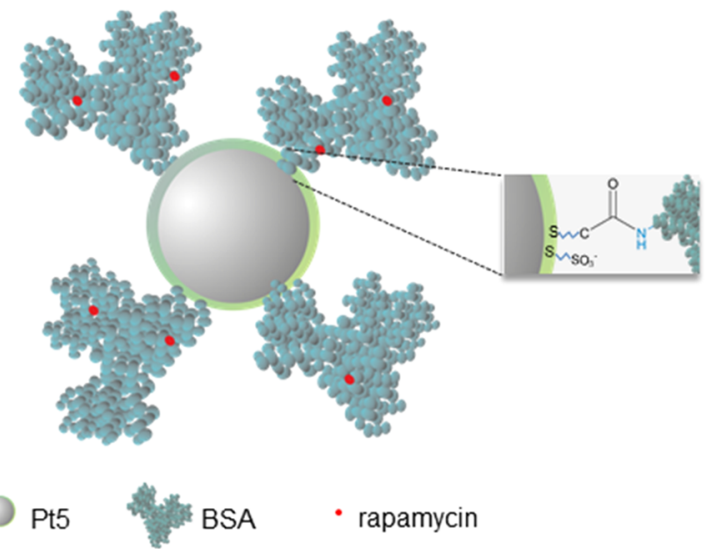

B

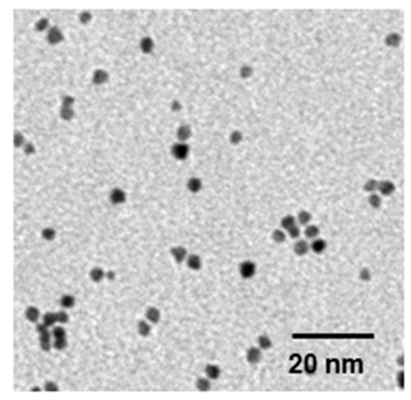

C

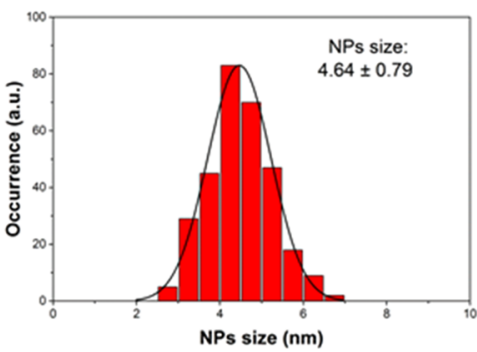

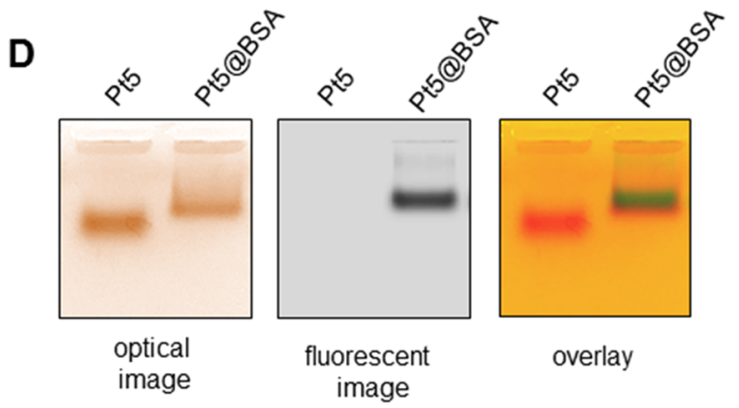

Figure 1. Characterization of Pt5@Rapa NPs. (A) Schematic representation of NP synthesis (features not to scale). (B) Representative transmission electron microscopy (TEM) image and (C) size distribution analysis of Pt5 NPs. (D) Characterization of Pt5 NPs functionalization by agarose gel electrophoresis. Left, optical image showing Pt5 NP retention in the agarose gel upon conjugation with fluorescent BSA. Middle, agarose gel analysis by fluorescent mode acquisition showing BSA conjugation. Right, overlay of optical and fluorescent acquisitions.

To address the possibility of developing a combinatorial treatment simultaneously targeting redox signaling and autophagic dysfunction, we developed a multifunctional nanocarrier, composed of rapamycin-functionalized platinum nanoparticles (PtNPs). This multifunctional nanocarrier combines the intrinsic antioxidant activity of $\mathrm{PtNPs}^{23-26}$ and the autophagy-stimulating activity of rapamycin. ${ }^{12}$ PtNPs are known to act as artificial antioxidant enzymes (nanozymes) and have proven strong antioxidant activity in cellular models of CCM disease, being able to restore their physiological ROS homeostasis. ${ }^{27}$ Moreover, the nanocarrier may allow the improvement of the cellular delivery of rapamycin, which is characterized by poor solubility in aqueous media, limiting its use as a free drug. ${ }^{28-30}$ Using established cellular models of CCM disease, we demonstrated that the combinatorial and synergistic pro-autophagic and antioxidant activities of the multifunctional nanocarrier were highly effective in rescuing major molecular and cellular phenotypes caused by KRIT1 loss-of-function, including defective autophagy, altered ROS homeostasis, and endothelial-to-mesenchymal transition $(\mathrm{EndMt})$, suggesting promising therapeutic applications.

\section{RESULTS AND DISCUSSION}

Preparation and Characterization of Platinum@BSARapamycin Nanocarrier (Pt5@Rapa NPs). The multifunctional nanocarrier was prepared using biocompatible, endotoxin-free, and highly catalytic citrate-capped PtNPs of $5 \mathrm{~nm}$, synthesized as previously reported. ${ }^{27}$ The radical scavenging activity of PtNPs was recently proved to restore intracellular redox homeostasis in a cellular model of CCM disease. ${ }^{27}$ Although the small size of these NPs may limit their drugloading capacity, their superior antioxidant nanozyme properties (due to their high surface-to-volume ratio) are particularly promising for the combinatorial treatment of $\mathrm{CCM}^{27}$ On the other hand, rapamycin was selected for its proven efficacy in rescuing defective autophagy in CCM cells. ${ }^{12}$ Because this 
A

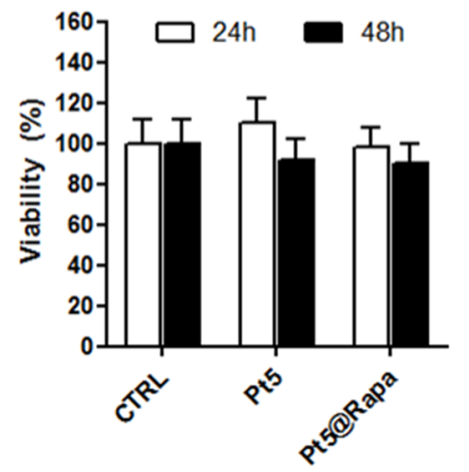

C

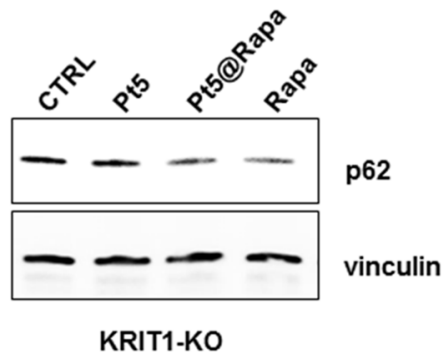

B

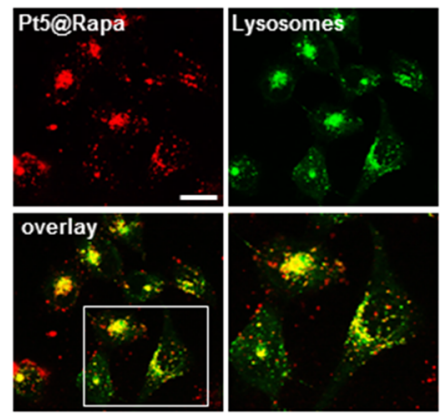

D

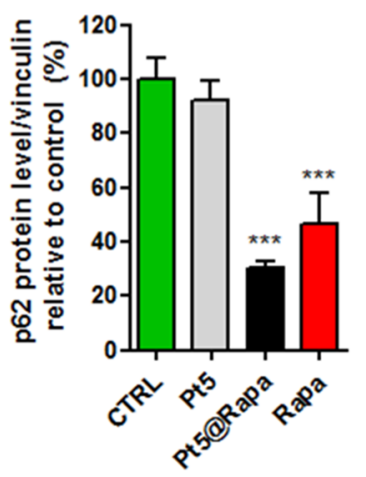

$\mathbf{E}$

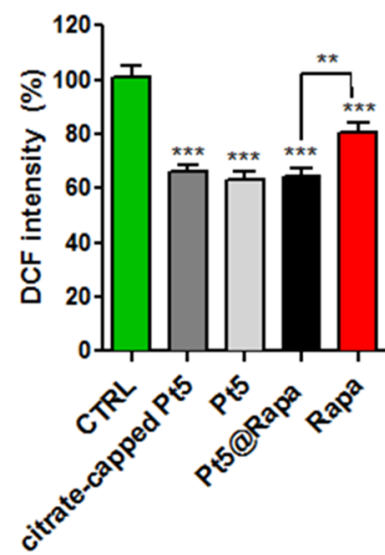

Figure 2. (A) Viability of KRIT1-KO MEFs after exposure for 24 and $48 \mathrm{~h}$ to $50 \mu \mathrm{g} / \mathrm{mL}$ Pt5 or Pt5@Rapa NPs. Viability of treated cells is expressed as relative to untreated control cells (CTRL). Data are reported as mean \pm standard deviation (SD). (B) Representative confocal fluorescence images of the internalization of Pt5@Rapa NPs into KRIT1-KO MEFs. Scale bar: $20 \mu \mathrm{m}$. Top left: fluorescent Pt5@Rapa NPs (red); top right: lysosomes stained with LysoTracker Green (green); low left: merged images; low right: magnification of the area in the white box. (C) Immunoblot analysis of p62 expression in KRIT1-KO MEFs untreated (CTRL) or treated for $15 \mathrm{~h}$ with $50 \mu \mathrm{g} / \mathrm{mL}$ Pt5, Pt5@Rapa NPs, or 500 nM rapamycin. Vinculin was used as a loading control. (D) Quantification of p62 on vinculin in KRIT1-KO MEFs, representative of three independent Western blot experiments, is reported. (E) ROS levels in KRIT1-KO MEFs untreated (CTRL), or exposed for $15 \mathrm{~h}$ to $50 \mu \mathrm{g} / \mathrm{mL}$ of citrate-capped Pt5, Pt5, Pt5@Rapa NPs, or $500 \mathrm{nM}$ rapamycin was evaluated by dichlorodihydrofluorescein diacetate (DCFH-DA) assay. DCF intensity of treated KRIT1-KO MEFs is expressed relative to untreated KRIT1-KO MEFs. At least three independent experiments were performed. Data are expressed as mean $\pm \mathrm{SD}$. Differences between treated samples and the control were considered statistically significant for $p$-values $<0.05(* * * p<$ $0.001)$.

drug has low bioavailability, ${ }^{29}$ we exploited Bovine Serum Albumin (BSA) to incorporate rapamycin within the hydrophobic pockets of the protein in the attempt to improve its solubilization and delivery, as well as to protect it from degradation. $^{31}$ Figure 1A illustrates our multifunctional platinum@BSA-rapamycin nanocarrier (Pt5@Rapa NPs). In particular, citrate-capped PtNPs were coated with two different thiols, one of them terminating with free carboxylic groups for the covalent binding of BSA through its amine groups, and the other one to provide colloidal stability to NPs during the bioconjugation procedure (see Materials and Methods). Exposed carboxyls were activated by click chemistry and covalently coupled to the primary amines of BSA via carbodiimide reaction. Drug loading was performed by two approaches: co-incubation of NPs with rapamycin and BSA or incubation of NPs with rapamycin-loaded BSA. These procedures gave similar results, provided that a low concentration of rapamycin is used to preserve the NP colloidal stability.

TEM analyses showed that Pt5 NPs were highly monodisperse (Figure 1B), with a size distribution centered around $5 \mathrm{~nm}$ (Figure 1C). The size and the dispersion degree of Pt5@Rapa NPs were investigated by Dynamic Light Scattering (DLS). An increase in the average hydrodynamic 
radius by $30 \mathrm{~nm}$ compared to $\mathrm{Pt} 5 \mathrm{NPs}$ was measured (Supporting Information Table S1). Binding of BSA on Pt5 NPs (Pt5@BSA) was confirmed by agarose gel electrophoresis (Figure 1D). Pt5@BSA NPs were retained in the agarose gel due to their higher molecular weight compared to nonfunctionalized Pt5 NPs, indicating the successful conjugation of the protein to the NPs. However, because BSA has molecular size comparable to our Pt5 NPs, only few BSA proteins can be conjugated to each NP. ${ }^{32}$ Actually, fluorescence analysis showed that each Pt5 NPs contained an average of 2 conjugated molecules of BSA. Furthermore, as probed by UV-vis, we estimated that each nanocarrier transported about two molecules of rapamycin. Although our nanovector had low drug loading capacity (few rapamycin molecules per NP), such bioconjugation conditions represented a good compromise between NP stability and monodispersion, drug loading, and high antioxidant activity, all crucial features for our combinatorial therapeutic approach.

Pt5@Rapa NPs for Rapamycin Delivery. To test the activity of the multifunctional rapamycin nanocarrier, we took advantage of KRIT1-knockout mouse embryonic fibroblast (MEF) cells (KRIT1-KO MEFs), an established cellular model of CCM disease characterized by impaired autophagy and high levels of intracellular ROS. ${ }^{12,16,17}$ This cellular model is well suited for testing combination approaches based on multifunctional nanocarriers. Several studies demonstrated that treatments of these cells with distinct compounds, endowed with antioxidant properties, were capable of recovering increased intracellular ROS levels and altered redox signaling, ${ }^{16,17,22,27,33}$ whereas their treatment with rapamycin was effective in rescuing also defective autophagy. ${ }^{12}$

We first assessed the toxicological profile of the Pt5@Rapa nanocarriers on KRIT1-KO MEF cells. Specifically, the cytocompatibility of Pt5@Rapa NPs was tested at concentrations up to $50 \mu \mathrm{g} / \mathrm{mL}$ for 24 and $48 \mathrm{~h}$, by WST-1 assay. Pt5@Rapa NPs were cytocompatible on MEFs (Figure 2A), indicating that Pt5 NP conjugation with rapamycin-loaded BSA does not alter their toxicological profile. Moreover, we assessed Pt5@Rapa NP cellular uptake and their intracellular fate by confocal microscopy. Confocal images showed an efficient internalization of Pt5@Rapa NPs and their compartmentalization within lysosomes (Figure 2B), confirming that NP uptake occurs by endocytosis, as previously demonstrated for nonfunctionalized citrate-capped $\mathrm{Pt} 5 \mathrm{NPs}{ }^{27}$ It has been demonstrated that also rapamycin-polymer conjugates are taken up by the endocytic pathway and compartmentalized within the lysosomes, where the release of the drug takes place. ${ }^{28}$ Accordingly, it is possible to speculate that, once endocytosed, the attack of peptidases to Pt5@Rapa NPs within lysosomes may induce the release of rapamycin from BSA, leading to rapamycin therapeutic action.

The efficacy of Pt5@Rapa NPs as rapamycin delivery system was tested by quantifying the expression of p62, a typical autophagic marker that accumulates when autophagy is inhibited. The total level of p62 was quantified in lysates of KRIT1-KO MEFs treated with Pt5 or Pt5@Rapa NPs. In parallel, cells were treated with free rapamycin, at a concentration known to restore the physiological p62 expression of wild-type cells (Supporting Information Figure S1). ${ }^{12}$ Western blot analysis revealed that the accumulation of p62 significantly decreased upon cell treatment with Pt5@ Rapa NPs (Figure 2C,D), indicating that rapamycin delivered by the nanocarrier is able to restore cell autophagy with similar efficiency as the free drug. On the contrary, no change in the level of p62 expression was detected in cells treated with nonfunctionalized Pt5 NPs, demonstrating that Pt5 NPs itself does not interfere with autophagy, unlike some reports with other metal NPs. ${ }^{34-36}$ These findings demonstrate that PtNPassisted rapamycin delivery is effective to elicit a therapeutic effect comparable to the free drug, even at a lower drug concentration. It can be hypothesized that rapamycin encapsulation within NPs might also have contributed to its efficacy by protecting it from the degradation and improving its internalization within the cells. Consistent with our findings, the use of rapamycin-loaded nanoparticles has been shown to target defective autophagy in muscular dystrophy and to enhance both skeletal muscle strength and cardiac contractile performance, which is not achievable with conventional oral rapamycin, even in pharmacological doses. ${ }^{37}$

Pt5@Rapa NPs as Antioxidant Nanocarriers. Citratecapped Pt5 NPs were recently found to restore ROS homeostasis in KRIT1-KO MEFs. ${ }^{27}$ To assess the ROS scavenging activity of Pt5@Rapa NPs, KRIT1-KO MEFs were treated with citrate-capped Pt5, Pt5, Pt5@Rapa NPs, or free rapamycin and ROS levels were quantified by the dichlorodihydrofluorescein diacetate (DCFH-DA) assay. Both Pt5 NPs and the multifunctional nanocarrier exhibited high ROS scavenging potential, which was comparable to that of nonfunctionalized citrate-capped Pt5 NPs, even though their surface is partially covered by thiols and BSA (Figure 2E). This suggests that the coating does not interfere with the catalytic reactions (as discussed above, it is likely that BSA molecules are degraded once in the lysosomal environment). Within the intracellular environment, Pt5@Rapa NPs exert significant antioxidant activity, directly reducing the ROS species, unlike free rapamycin that indirectly regulates the ROS levels by acting on autophagy pathways that control the clearance of ROS-generating dysfunctional mitochondria. ${ }^{38}$ Therefore, the drug itself has a weak antioxidant activity. Consistently, additive effect of rapamycin within the nanovector was not detectable, probably due to its lower concentration on the nanocarrier compared to that of the free drug. These results demonstrated that our nanocarrier is also an efficient modulator of ROS homeostasis in a cellular model of CCM disease, confirming its potential as multifunctional nanovector for combination therapy.

Pt5@Rapa NPs as Angiogenesis Modulator. CCM proteins play a major role in the maintenance of endothelial cell-cell junction stability and blood-brain barrier (BBB) integrity through the tight modulation of the interplay between autophagy and redox homeostasis and signaling. ${ }^{10,14,15,39-43}$ By impairing autophagy and dysregulating ROS homeostasis, lossof-function of CCM genes, including KRIT1, causes EndMt, ${ }^{12,20,44}$ a process whereby endothelial cells lose their specific markers and cell-cell contacts, weakening their barrier function. ${ }^{45}$ In light of this mechanism, we tested the efficacy of our multifunctional nanocarrier to attenuate or reverse EndMt and re-establish physiological angiogenesis of KRIT1-depleted endothelial cells, comparing its effect with that of rapamycin alone or PtNPs. Once verified the cytocompatibility of Pt5 NPs on wild-type primary Human Umbelical Vein Endothelial Cells (HUVEC) (Supporting Information Figure S2), the effect of Pt5 NPs, rapamycin, and Pt5@Rapa NPs was evaluated on unsilenced and KRIT1-silenced HUVECs (KRIT1-KO HUVECs) by an in vitro angiogenesis assay (Figure 3A). The formation of a network of capillary-like 
A

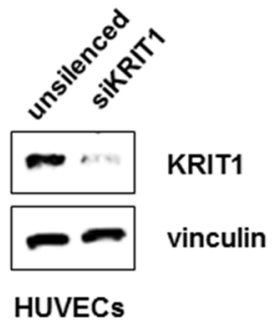

B

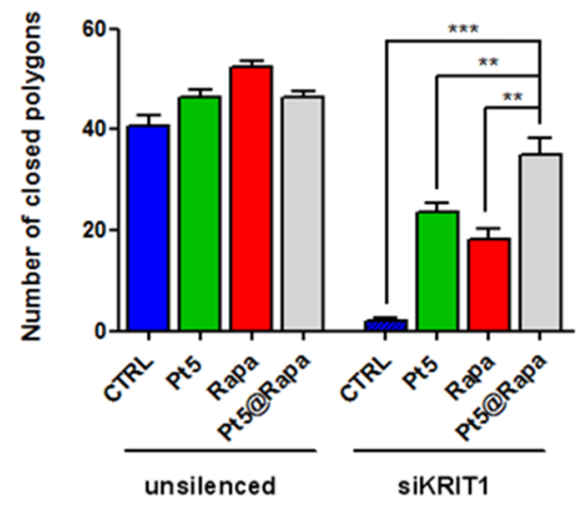

siKRIT1
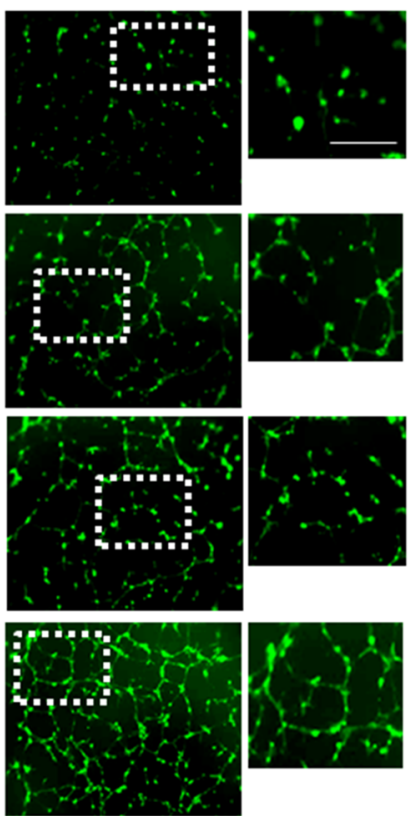

Figure 3. (A) Immunoblot analysis of KRIT1 silencing in HUVECs transfected with negative control siRNA (unsilenced) or KRIT1 siRNA (siKRIT1) for $72 \mathrm{~h}$. Vinculin was used as loading control. (B) Quantitative evaluation of tube formation as the number of closed polygons formed in 8 fields for each experimental condition: unsilenced or KRIT1-silenced HUVECs, left untreated (CTRL) or treated for $15 \mathrm{~h}$ with $50 \mu \mathrm{g} / \mathrm{mL}$ of Pt5 NPs, $500 \mathrm{nM}$ rapamycin, or $50 \mu \mathrm{g} / \mathrm{mL}$ of Pt5@Rapa NPs. All the data are presented as mean $\pm \mathrm{SD}$. (C) Representative images of one of three independent experiments of capillary networks visualized by fluorescent calcein staining. Magnifications are reported in the inserts. Scale bars, 200 $\mu \mathrm{m}$ for full fields and $400 \mu \mathrm{m}$ for magnified fields.

structures on Matrigel was followed over a period of $6-12 \mathrm{~h}$ after cellular exposure for $15 \mathrm{~h}$ to treatments (Figure 3B,C). Normal angiogenesis was observed in all unsilenced cells, regardless of the treatments. Conversely, KRIT1 knockdown completely impaired cell ability to form capillary-like tube structures. This is in agreement with previous reports showing that endothelial cells with the loss-of-function of CCM genes fail to organize into well-formed capillary-like networks. ${ }^{46}$ Interestingly, KRIT1-KO HUVECs exposed to Pt5 NPs developed some capillary-like structures, demonstrating that PtNPs can promote the partial recovery of the endothelial phenotype of KRIT1-silenced HUVECs. This might be attributed to the intrinsic property of Pt5 NPs to act as antioxidant nanozymes, being ROS homeostasis crucial for normal endothelial cell function and signaling. ${ }^{47}$ As expected, also rapamycin treatment was found to be effective in reactivating some in vitro angiogenesis. Indeed, treatment with rapamycin was previously demonstrated to reverse EndMt by increasing the expression of key endothelial cell markers in KRIT1-KO endothelial cells. ${ }^{12}$ However, neither the antioxidant treatment with bare nanoparticles nor autophagy induction with rapamycin alone was sufficient to fully inhibit EndMt and restore physiological in vitro angiogenesis, further supporting the requirement of a combined treatment with antioxidants and autophagy inducers. Remarkably, KRIT1-KO HUVECs treated with Pt5@Rapa NPs were able to undergo in vitro angiogenesis almost comparably to unsilenced cells, thus demonstrating the synergistic effect of the combination of the two therapeutic activities.

A possible model of the molecular mechanism of Pt5@Rapa NPs in KRIT1-KO endothelial cells is reported in Figure 4C. Specifically, the loss-of-function of KRIT1 strongly affects autophagy, leading to the aberrant accumulation of p62 and causing an increase in intracellular ROS and the EndMt switch 


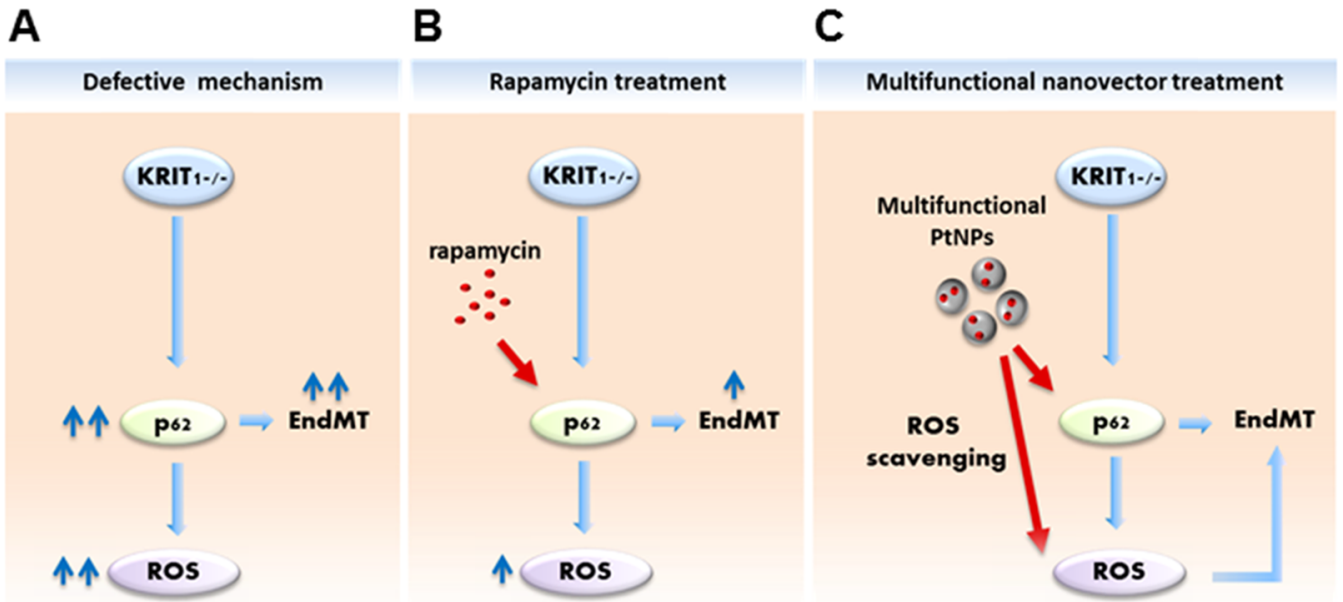

Figure 4. Schematic model of Pt5@Rapa NP mechanism of action in KRIT1-KO cells. (A) KRIT1 loss-of-function impairs autophagy, leading to the aberrant accumulation of autophagy adapter p62, which, in turn, enhances intracellular ROS and promotes the EndMt switch, the two crucial events that contribute to CCM progression. (B) Therapeutic reactivation of autophagy with rapamycin in KRIT1-KO cells decreases p62 accumulation, reduces intracellular accumulation of ROS, and inhibits the EndMt switch. (C) PtNPs-based combinatorial treatment exerts synergistic effects that enhances the efficacy of the therapeutic treatment. Pt5@Rapa NPs in KRIT1-KO cells work as multifunctional nanoplatform simultaneously acting as ROS-scavenging materials and drug nanocarriers. Pt5@Rapa NPs counterbalance the increase in intracellular ROS levels through their activity of antioxidant enzymes and deliver rapamycin into the cells to decrease the aberrant accumulation of p62. Together, these activities restore ROS homeostasis and inhibit EndMt with a higher efficacy than the treatment with rapamycin alone.

(Figure 4A). Cell treatment with rapamycin reactivates autophagy, thereby decreasing p62 accumulation, partially reducing the ROS levels and reverting the EndMt switch (Figure 4B). On the other hand, given the intrinsic ROSscavenging activity of the Pt5 NPs core and the pro-autophagic activity of the conjugated rapamycin, Pt5@Rapa NPs can directly counteract both increased intracellular ROS levels and defective autophagy, thus exerting synergistic effects that efficiently limit the major molecular and cellular dysfunctions associated with defective autophagy, including ROS overproduction and EndMt. Such a combined action allows endothelial cells to reacquire their specific phenotype and function (Figure 4C). Hence, the synergism of Pt5@Rapa NPs demonstrates the superiority of the combinatorial approach over the targeting of single molecular pathways, suggesting that NP-based combinatorial therapies may represent a promising strategy to treat CCM disease. Furthermore, it is also possible to speculate that the leaky/abnormal nature of CCM vasculature would favor NP-based drug delivery in vivo because NPs may accumulate at the level of the lesions through the enhanced permeability and retention phenomenon rather than penetrate through tight endothelial junctions of normal blood vessels of other tissues, minimizing systemic side effects and toxicity of the free drug. ${ }^{48}$ In general, given their superior performances over classical antioxidant compounds in terms of long-term stability, prolonged action, and wider operational conditions, ${ }^{26,27}$ the multifunctional PtNPs developed in this work could be adapted to targeted combination therapy approaches for other oxidative stress-related diseases.

\section{CONCLUSIONS}

In recent years, nanocarriers have emerged as a promising tool for the co-delivery of multiple drugs for the combination therapy of complex diseases. Integrating multiple drugs in a single nanocarrier has the advantages of beneficially overlapping the pharmacological profiles of drugs, reduced toxicity, and ratiometric drug delivery and offers the possibility to achieve maximum therapeutic synergy and personalized treatments. ${ }^{49}$ Here, we exploited the nanozyme properties and the versatile surface modification of PtNPs to engineer a multifunctional drug delivery system with combined antioxidant properties and autophagy-stimulating activity to prove their therapeutic potential in combinatorial treatments. The CCM cellular model represented a suitable benchmark to demonstrate the ability of the multifunctional nanovector to work simultaneously as antioxidant nanozyme and drug nanocarrier and the superiority of the combinatorial treatment over single treatments with PtNPs or the free drug alone in rescuing major phenotypic hallmarks of cellular models of CCM disease. Our proof-of-concept study also suggests that PtNPs possess suitable features to be considered for further investigations as multifunctional nanocarriers in combinatorial treatments of CCM or other oxidative stress-related complex diseases. The development of advanced delivery systems, including those based on pickering emulsions, ${ }^{50}$ could be envisaged to attempt possible in vivo translation of the proposed nanovector.

\section{MATERIALS AND METHODS}

Synthesis of Pt5 NPs. Immediately after the synthesis of the citrate-capped Pt5 NPs, ${ }^{27} 0.8 \mathrm{~mL}$ of a solution $50 \mathrm{mM}$ sodium 3-mercapto-1-propanesulfonate (Sigma-Aldrich), and 2 $\mathrm{mL}$ of a solution $20 \mathrm{mM}$ of 3-mercaptopropionic acid, MPA (Sigma-Aldrich), were sequentially added to the reaction mixture. The solution was kept under vigorous stirring for at least $1 \mathrm{~h}$ at $80{ }^{\circ} \mathrm{C}$. Thiol molecules form a self-assembled monolayer on the citrate-capped Pt5 NPs due to the high affinity of thiols for noble metals ${ }^{51-56}$ and the strong Pt-sulfur interaction. ${ }^{57,58}$ Afterward, the solution was cooled to room temperature, washed with Milli-Q water using $50 \mathrm{~K}$ Amicon Ultra centrifugal filters, and stored under $4{ }^{\circ} \mathrm{C}$ for future experiments. Pt5 NPs were then characterized by dynamic light scattering (DLS) spectroscopy using a Zetasizer Nano ZS 
System equipped with a $633 \mathrm{~nm} \mathrm{He}-\mathrm{Ne}$ laser (Malvern Instruments).

TEM Imaging of Pt5 NPs. The shape and the size of Pt5 NPs were analyzed using the TEM analysis. A dilute solution of PtNPs dispersed in methanol was deposited on a carboncoated grid and left to dry in an oven. The TEM images of NPs were recorded by using a JEOL JEM 1011 microscope. The size of PtNPs was determined by ImageJ software, measuring the diameter of more than 300 NPs.

Preparation of Platinum@BSA-Rapamycin Nanocarrier (Pt5@Rapa NPs). BSA conjugation with Pt5 NPs was carried out by a covalent coupling of the carboxylic groups of the MPA layer with the primary amines of BSA (SigmaAldrich) via carbodiimide chemistry. $\mathrm{N}$-(3-dimethylaminopropyl)- $N$-ethylcarbodiimide hydrochloride (EDC) (SigmaAldrich, BioXtra) and N-hydroxysuccinimide (NHS) (SigmaAldrich) were used as cross-linking agents. EDC was used to mediate the formation of peptide linkages between BSA and MPA, and NHS for improving the reaction rate and efficiency. ${ }^{59}$

Activation of Pt5 NPs was carried out using EDC and NHS as follows: $15 \mu \mathrm{L}$ of a solution $30 \mathrm{mg} / \mathrm{mL}$ of EDC and $15 \mu \mathrm{L}$ of a solution $40 \mathrm{mg} / \mathrm{mL}$ of NHS were added to $1 \mathrm{~mL}$ of a solution $300 \mu \mathrm{g} / \mathrm{mL}$ of Pt5 NPs. The reaction mixture was gently shaken for $5 \mathrm{~min}$ at room temperature. The excess of EDC/NHS was immediately removed from the NP solution by centrifugation at $15000 \mathrm{rcf}$ for $30 \mathrm{~min}$. Afterward, the supernatant was collected in another vial, the pellet was resuspended in Milli-Q water, and both the solutions were centrifuged again. This washing procedure was repeated two more times. The NP solution was then resuspended into $1 \mathrm{~mL}$ of PBS.

Pt5@Rapa nanovector was obtained by adding simultaneously $100 \mu \mathrm{L}$ of a solution of $2 \mathrm{mg} / \mathrm{mL}$ of BSA and $20 \mu \mathrm{L}$ of a solution $100 \mu \mathrm{M}$ of rapamycin (CalbioChem) in dimethyl sulfoxide to $1 \mathrm{~mL}$ of EDC/NHS-activated NPs. The solution was gently shaken and incubated for $2 \mathrm{~h}$ at room temperature. The resultant Pt5@Rapa NPs were washed twice with PBS at $15000 \mathrm{rcf}$ for $30 \mathrm{~min}$ to remove unconjugated BSA and drug molecules. The hydrodynamic diameter of PtNPs was then measured by DLS spectroscopy to confirm the successful bioconjugation. For fluorescence analysis, BSA-conjugated PtNPs were prepared using Alexa Fluor 647 conjugated BSA (A34785, Thermofisher).

Gel Electrophoresis of Platinum@BSA NPs. Gel electrophoresis was carried out for $20 \mathrm{~min}$ at $70 \mathrm{~V}$ on $0.6 \%$ agarose gel using sodium boric acid buffer $\mathrm{pH}$ 8.5. Ten microliters of two solutions containing 50\% glycerol and $\mathrm{Pt} 5$ or Pt5 NPs conjugated to fluorescent BSA was loaded in each well. Images were taken with Typhoon Trio (GE).

Characterization of Pt5@Rapa NPs. The number of BSA molecules per PtNP has been quantified by fluorescence analysis comparing the results with a fluorescent BSA standard calibration curve. A Infinite 200 Pro Tecan microplate reader was used for reading the BSA fluorescence intensity, setting the excitation filter at $647 \mathrm{~nm}$ and the emission filter at $680 \mathrm{~nm}$.

The evaluation of the amount of rapamycin per nanovector has been obtained indirectly by considering the number of BSA per nanovector and their ability to load rapamycin. Rapamycin-loading capacity of each BSA protein was calculated using UV-vis measurements. BSA and rapamycin were incubated at equimolar ratio for the nanovector synthesis. The amount of rapamycin that does not get trapped within
BSA is able to pass through the $3 \mathrm{~K}$ Amicon Ultra centrifugal filters and then can be measured by UV-vis.

Cell Culture. Mouse embryonic fibroblasts (MEFs) ${ }^{16}$ were cultivated in Dulbecco's modified Eagle's medium (DMEM) (Sigma-Aldrich) supplemented with $10 \%(\mathrm{v} / \mathrm{v})$ fetal bovine serum (Sigma-Aldrich), $100 \mathrm{U} / \mathrm{mL}$ penicillin, and $100 \mathrm{mg} / \mathrm{mL}$ streptomycin (Sigma-Aldrich). Human Umbilical Vein Endothelial Cells (HUVECs) were cultivated on $0.1 \%$ gelatin (Sigma-Aldrich) in Medium 200 (Cascade BiologicsInvitrogen cell culture, Cat. no. M-200-500) supplemented with Low Serum Growth supplement kit (Cascade BiologicsInvitrogen cell culture, Cat. no. S-003-K). Cells were incubated at $37{ }^{\circ} \mathrm{C}$ under a humidified controlled atmosphere with $5 \%$ $\mathrm{CO}_{2}$. The HUVECs were used for experiments between passages 2 and 6 .

WST-1 Assay. In vitro toxicological profile of Pt5 and Pt5@Rapa NPs on MEFs and HUVECs was evaluated by WST-1 assay (Sigma-Aldrich) according to the previously described method. ${ }^{60}$ Briefly, MEFs (25000 cells/well) were plated in a 96-well tissue culture plate in a final volume of 100 $\mu \mathrm{L}$. After $24 \mathrm{~h}$ of adhesion, the culture medium was removed and replaced with medium containing Pt5 or Pt5@Rapa NPs at a concentration of $50 \mu \mathrm{g} / \mathrm{mL}$, up to 24 or $48 \mathrm{~h}$. Afterward, the cells were washed three times with PBS and incubated for 1 $\mathrm{h}$ with medium containing 10\% WST. The HUVECs (50 000 cells/well) were plated in a 96-well tissue culture plate in a final volume of $100 \mu \mathrm{L}$. After $24 \mathrm{~h}$ of adhesion, the culture medium was removed and replaced with medium containing a series of Pt5 NPs dilutions ranging from 10 to $50 \mu \mathrm{g} / \mathrm{mL}$, and the cells were cultured for other $24 \mathrm{~h}$. Afterward, the cells were washed three times with PBS and incubated for $1 \mathrm{~h}$ with a medium containing 10\% WST. An Infinite 200 Pro Tecan microplate reader was used for reading the cell viability results.

DCFH-DA Assay. MEFs (25000 cells/well) were plated in 96-well microplates (Constar) and exposed to $50 \mu \mathrm{g} / \mathrm{mL}$ of citrate-capped Pt5, Pt5, Pt5@Rapa NPs, and $500 \mathrm{nM}$ rapamycin. After $15 \mathrm{~h}$, the cells were washed three times with PBS with $\mathrm{Ca}^{2+}$ and $\mathrm{Mg}^{2+}$ and incubated at $37{ }^{\circ} \mathrm{C}$ for 10 min with $5 \mu \mathrm{M}$ DCFH-DA (Sigma) in PBS. An Infinite 200 Pro Tecan microplate reader was used for reading the DCF fluorescence intensity, setting the excitation filter at $480 \mathrm{~nm}$ and the emission filter at $520 \mathrm{~nm}$. The results were normalized with respect to untreated control cells.

Cell Transfection and siRNA-Mediated Gene Silencing. For KRIT1 knockdown with small interfering RNA, the HUVECs were transfected twice with a mix of $4 \times$ predesigned iBONi siRNA against KRIT1 (D-00101-Plus, Ribbox GmbH, Germany) or an iBONi siRNA negative control (K-003010005-N3, Ribbox GmbH). HiPerFect Transfection Reagent (Qiagen) was used according to manufacturer's instructions. Briefly, on the day of transfection, the HUVECs $\left(2 \times 10^{5}\right.$ cells well) were transfected in 6-well dishes with negative control siRNA or with siRNA against KRIT1 at the final concentration of $120 \mathrm{nM}$ (30 nM each siRNA). The siRNA transfection procedure was repeated after $24 \mathrm{~h}$ to enhance KRIT1 knockdown efficiency. KRIT1 silencing was monitored by Western blotting analysis.

Western Blotting. The transfected HUVECs were lysed in radioimmunoprecipitation assay buffer containing protease and phosphatase inhibitor cocktails (Sigma-Aldrich) for $20 \mathrm{~min}$ on ice. After centrifugation at $14000 \mathrm{rpm}$ for $10 \mathrm{~min}$ at $4{ }^{\circ} \mathrm{C}$, the proteins were quantified by Micro BCA Protein Assay kit (Thermo Fisher Scientific). Total cell lysates containing equal 
amounts of total proteins $(20 \mu \mathrm{g})$ were separated by $10 \%$ miniprotean TGX precast acrylamide gels (Bio-rad Laboratories) and electroblotted onto poly(vinylidene difluoride) (PVDF) membranes (Bio-rad Laboratories).

MEFs (500000 cells/well) treated for $15 \mathrm{~h}$ with $50 \mu \mathrm{g} / \mathrm{mL}$ of Pt5, Pt5@Rapa NPs, or 500 nM rapamycin were lysed in a buffer containing $50 \mathrm{mM}$ Tris $\mathrm{pH} 8.0,150 \mathrm{mM} \mathrm{NaCl}, 10 \%$ glycerol, $1 \%$ Triton X-100, 1\% sodium dodecyl sulfate, protease, and phosphatase inhibitor cocktails (Sigma-Aldrich) for $20 \mathrm{~min}$ on ice. After centrifugation at $14000 \mathrm{rpm}$ for 10 $\min$ at $4{ }^{\circ} \mathrm{C}$, the proteins were quantified by Micro BCA Protein Assay kit (Thermo Fisher Scientific). Total cell lysates containing equal amounts of total proteins $(5 \mu \mathrm{g})$ were separated by $4-20 \%$ mini-protean TGX precast acrylamide gels (Bio-Rad Laboratories) and electroblotted onto PVDF membranes (Bio-Rad Laboratories). The blots were blocked with 5\% nonfat milk (Sigma-Aldrich) in Tris-buffered saline (TBS) containing $0.05 \%$ Tween 20 for $1 \mathrm{~h}$ at room temperature and incubated overnight at $4{ }^{\circ} \mathrm{C}$ with primary antibodies diluted in TBS $1 \%$ BSA. The membranes were then washed three times with TBS $0.3 \%$ Tween 20 and incubated with appropriate horseradish peroxidase-conjugated secondary antibodies (1:2500) in TBS $0.3 \%$ Tween 20 for $1 \mathrm{~h}$ at room temperature. The proteins were visualized according to manufacturer's instruction by an enhanced chemiluminescence detection system (Bio-Rad) with Typhoon Trio (GE). Protein band intensity was quantified by using ImageJ software (NIH).

Antibodies. Rabbit polyclonal anti-P62/SQSTM1 antibody (P0067, Sigma-Aldrich), rabbit recombinant monoclonal KRIT1 antibody [EPR16560] (ab196025, Abcam), mouse monoclonal anti-vinculin antibody (V9264, Sigma-Aldrich), and goat anti-mouse and anti-rabbit $\operatorname{IgG}(\mathrm{H}+\mathrm{L})$-HRP conjugated secondary antibodies (1706515, 1706515, BioRad) were used.

Confocal Microscopy. MEFs (10000 cells/well) cells were seeded in an 8-well chamber (VWR) and incubated at 37 ${ }^{\circ} \mathrm{C}$ and $5 \% \mathrm{CO}_{2}$. After $24 \mathrm{~h}$, the cellular medium was removed and replaced with $50 \mu \mathrm{g} / \mathrm{mL}$ Pt5@Rapa NPs containing Alexa Fluor 647 conjugated BSA diluted in fresh FluoroBrite DMEM medium (Thermo Fisher Scientific). After $30 \mathrm{~min}$, the cells were imaged with Leica TCS SP8 confocal microscope using a $63 \times$ oil immersion objective. Pt5@Rapa NPs were imaged using a $647 \mathrm{~nm}$ excitation wavelength. For lysosomes imaging, the cells were incubated with $75 \mathrm{nM}$ LysoTracker Green DND-26 (Molecular probes). After 10 min of incubation, the medium was removed, cells were washed three times with PBS, and imaged using a $488 \mathrm{~nm}$ excitation wavelength for LysoTracker Green signal detection.

In Vitro Angiogenesis Assay. Seventy-two hours after transfection with negative control siRNA or $4 \times$ predesigned KRIT1 siRNA, the HUVECs were incubated with $50 \mu \mathrm{g} / \mathrm{mL}$ Pt5, Pt5@Rapa NPs, or 500 nM rapamycin. After 15 h, the In Vitro Angiogenesis Assay (Millipore) was performed following the manufacturer's instructions. Briefly, untreated or treated HUVECs $\left(10 \times 10^{3}\right)$ were plated in each well of a 96-well plate precoated with $50 \mu \mathrm{L}$ of ECMatrix (Millipore) in complete Medium 200. After $4 \mathrm{~h}$ of incubation, the samples were stained with Calcein AM (Thermo Fisher Scientific) for $20 \mathrm{~min}$ at $37^{\circ} \mathrm{C}$ and the formation of capillary-like structures was analyzed under an EVOS Cell Imaging microscope (Thermo Fisher Scientific) at $4 \times$ magnification. To evaluate the degree of angiogenesis progression, the number of closed polygons formed in eight replicates was counted using Image J software (NIH).

Statistical Analysis. The data are expressed as mean \pm SD. For statistical analysis, GraphPad Prism 5 statistical analysis software was used (GraphPad Prism version 5.00 for Windows, GraphPad Prism 5 Software, San Diego, California). The $p$-values were calculated using one-way analysis of variance and compared to the corresponding control by the Bonferroni post-test. Each experiment was independently performed at least three times. Differences between treated samples and controls were considered statistically significant for $p$-values $<0.05$. $(* p<0.05, * * p<0.01$, and $* * * p<0.001)$.

\section{ASSOCIATED CONTENT}

\section{Supporting Information}

The Supporting Information is available free of charge on the ACS Publications website at DOI: 10.1021/acsomega.8b01653.

DLS data of Pt5 and Pt5@Rapa NPs (Table S1); treatment of KRIT1-KO MEFs with rapamycin restores p62 expression to the levels of wild-type MEFs (Figure S1); cytocompatibility of Pt5 NPs on HUVECs (Figure S2) (PDF)

\section{AUTHOR INFORMATION}

\section{Corresponding Authors}

*E-mail: francesco.retta@unito.it. Web: www.ccmitalia.unito.it (S.F.R.).

*E-mail: pierpaolo.pompa@iit.it (P.P.P.).

ORCID

Mauro Moglianetti: 0000-0003-0747-7963

Saverio Francesco Retta: 0000-0001-9761-2959

Pier Paolo Pompa: 0000-0001-7549-0612

\section{Funding}

This work was supported by the Telethon Foundation (grant GGP15219 to S.F.R.).

\section{Notes}

The authors declare no competing financial interest.

\section{ACKNOWLEDGMENTS}

The authors are deeply grateful to Simona Delle Monache, Saverio Marchi, and Paolo Pinton for providing useful reagents, helpful discussion, and critical revision of the manuscript. Moreover, they acknowledge the Italian Research Network for Cerebral cavernous malformation (CCM Italia, http://www.ccmitalia.unito.it) and the Associazione Italiana Angiomi Cavernosi (AIAC, http://www.ccmitalia.unito.it/ aiac) for fundamental collaboration and support, and Luca Goitre, Eliana Trapani, Valerio Benedetti, Claudia Fornelli, Alessia Zotta, Federica Geddo, Giulia Costantino, Giovanna Bratti, Gaudenzio Inverso, and Santina Barbaro for helpful discussion.

\section{ABBREVIATIONS}

BSA, bovine serum albumin; CCM, cerebral cavernous malformation; EndMt, endothelial-to-mesenchymal transition; PtNPs, platinum nanoparticles; Pt5 NPs, thiol-coated PtNPs of 5 nm; Pt5@Rapa NPs, platinum@BSA-rapamycin nanoparticles; ROS, reactive oxygen species 


\section{REFERENCES}

(1) Chen, S.-h.; Lahav, G. Two is better than one; toward a rational design of combinatorial therapy. Curr. Opin. Struct. Biol. 2016, 41, 145-150.

(2) Ravi, I. Complex diseases require complex therapies. EMBO Rep. 2013, 14, 1039-1042.

(3) Woodcock, J.; Griffin, J. P.; Behrman, R. E. Development of Novel Combination Therapies. N. Engl. J. Med. 2011, 364, 985-987.

(4) He, B.; Lu, C.; Zheng, G.; He, X.; Wang, M.; Chen, G.; Zhang, G.; Lu, A. Combination therapeutics in complex diseases. J. Cell. Mol. Med. 2016, 20, 2231-2240.

(5) Batra, S.; Lin, D.; Recinos, P. F.; Zhang, J.; Rigamonti, D. Cavernous malformations: natural history, diagnosis and treatment. Nat. Rev. Neurol. 2009, 5, 659-670.

(6) Fontanella, M.; Bacigaluppi, S. Treatment of cerebral cavernous malformations: where do we stand? J. Neurosurg. Sci. 2015, 59, 199200.

(7) Rigamonti, D. Cavernous Malformations of the Nervous System; Cambridge University Press: Cambridge, 2011.

(8) Gault, J.; Sarin, H.; Awadallah, N. A.; Shenkar, R.; Awad, I. A. Pathobiology of Human Cerebrovascular Malformations: Basic Mechanisms and Clinical Relevance. Neurosurgery 2004, 55, 1-17.

(9) Choquet, H.; Pawlikowska, L.; Lawton, M. T.; Kim, H. Genetics of Cerebral Cavernous Malformations: Current Status and Future Prospects. J. Neurosurg. Sci. 2015, 59, 211-220.

(10) Retta, S. F.; Glading, A. J. Oxidative stress and inflammation in cerebral cavernous malformation disease pathogenesis: Two sides of the same coin. Int. J. Biochem. Cell Biol. 2016, 81, 254-270.

(11) Trapani, E.; Retta, S. F. Cerebral cavernous malformation (CCM) disease: from monogenic forms to genetic susceptibility factors. J. Neurosurg. Sci. 2015, 59, 201-209.

(12) Marchi, S.; Corricelli, M.; Trapani, E.; Bravi, L.; Pittaro, A.; Delle Monache, S.; Ferroni, L.; Patergnani, S.; Missiroli, S.; Goitre, L.; Trabalzini, L.; Rimessi, A.; Giorgi, C.; Zavan, B.; Cassoni, P.; Dejana, E.; Retta, S. F.; Pinton, P. Defective autophagy is a key feature of cerebral cavernous malformations. EMBO Mol. Med. 2015, 7, 14031417.

(13) Marchi, S.; Retta, S. F.; Pinton, P. Cellular processes underlying cerebral cavernous malformations: Autophagy as another point of view. Autophagy 2016, 12, 424-425.

(14) Marchi, S.; Trapani, E.; Corricelli, M.; Goitre, L.; Pinton, P.; Retta, S. F. Beyond multiple mechanisms and a unique drug: Defective autophagy as pivotal player in cerebral cavernous malformation pathogenesis and implications for targeted therapies. Rare Dis. 2016, 4, No. e1142640.

(15) Whitehead, K. J.; Chan, A. C.; Navankasattusas, S.; Koh, W.; London, N. R.; Ling, J.; Mayo, A. H.; Drakos, S. G.; Jones, C. A.; Zhu, W.; Marchuk, D. A.; Davis, G. E.; Li, D. Y. The cerebral cavernous malformation signaling pathway promotes vascular integrity via Rho GTPases. Nat. Med. 2009, 15, 177-184.

(16) Goitre, L.; Balzac, F.; Degani, S.; Degan, P.; Marchi, S.; Pinton, P.; Retta, S. F. KRIT1 Regulates the Homeostasis of Intracellular Reactive Oxygen Species. PLoS One 2010, 5, No. e11786.

(17) Goitre, L.; De Luca, E.; Braggion, S.; Trapani, E.; Guglielmotto, M.; Biasi, F.; Forni, M.; Moglia, A.; Trabalzini, L.; Retta, S. F. KRIT1 loss of function causes a ROS-dependent upregulation of c-Jun. Free Radical Biol. Med. 2014, 68, 134-147.

(18) McDonald, D. A.; Shi, C.; Shenkar, R.; Stockton, R. A.; Liu, F.; Ginsberg, M. H.; Marchuk, D. A.; Awad, I. A. Fasudil Decreases Lesion Burden in a Murine Model of Cerebral Cavernous Malformation Disease. Stroke 2012, 43, 571-574.

(19) Gibson, C. C.; Zhu, W.; Davis, C. T.; Bowman-Kirigin, J. A.; Chan, A. C.; Ling, J.; Walker, A. E.; Goitre, L.; Delle Monache, S.; Retta, S. F.; Shiu, Y.-T. E.; Grossmann, A. H.; Thomas, K. R.; Donato, A. J.; Lesniewski, L. A.; Whitehead, K. J.; Li, D. Y. Strategy for Identifying Repurposed Drugs for the Treatment of Cerebral Cavernous Malformation. Circulation 2015, 131, 289-299.

(20) Bravi, L.; Malinverno, M.; Pisati, F.; Rudini, N.; Cuttano, R.; Pallini, R.; Martini, M.; Larocca, L. M.; Locatelli, M.; Levi, V.; Bertani,
G. A.; Dejana, E.; Lampugnani, M. G. Endothelial Cells Lining Sporadic Cerebral Cavernous Malformation Cavernomas Undergo Endothelial-to-Mesenchymal Transition. Stroke 2016, 47, 886-890.

(21) Goitre, L.; DiStefano, P. V.; Moglia, A.; Nobiletti, N.; Baldini, E.; Trabalzini, L.; Keubel, J.; Trapani, E.; Shuvaev, V. V.; Muzykantov, V. R.; Sarelius, I. H.; Retta, S. F.; Glading, A. J. Up-regulation of NADPH oxidase-mediated redox signaling contributes to the loss of barrier function in KRIT1 deficient endothelium. Sci. Rep. 2017, 7, No. 8296.

(22) Moglia, A.; Goitre, L.; Gianoglio, S.; Baldini, E.; Trapani, E.; Genre, A.; Scattina, A.; Dondo, G.; Trabalzini, L.; Beekwilder, J.; Retta, S. F. Evaluation of the bioactive properties of avenanthramide analogs produced in recombinant yeast. BioFactors 2015, 41, 15-27.

(23) Fan, J.; Yin, J.-J.; Ning, B.; Wu, X.; Hu, Y.; Ferrari, M.; Anderson, G. J.; Wei, J.; Zhao, Y.; Nie, G. Direct evidence for catalase and peroxidase activities of ferritin-platinum nanoparticles. Biomaterials 2011, 32, 1611-1618.

(24) Zhang, L.; Laug, L.; Münchgesang, W.; Pippel, E.; Gösele, U.; Brandsch, M.; Knez, M. Reducing Stress on Cells with ApoferritinEncapsulated Platinum Nanoparticles. Nano Lett. 2010, 10, 219-223.

(25) Hirakawa, K.; Sano, S. Platinum Nanoparticle Catalyst Scavenges Hydrogen Peroxide Generated from Hydroquinone. Bull. Chem. Soc. Jpn. 2009, 82, 1299-1303.

(26) Pedone, D.; Moglianetti, M.; De Luca, E.; Bardi, G.; Pompa, P. P. Platinum nanoparticles in nanobiomedicine. Chem. Soc. Rev. 2017, 46, 4951-4975.

(27) Moglianetti, M.; De Luca, E.; Pedone, D.; Marotta, R.; Catelani, T.; Sartori, B.; Amenitsch, H.; Retta, S. F.; Pompa, P. P. Platinum nanozymes recover cellular ROS homeostasis in an oxidative stress-mediated disease model. Nanoscale 2016, 8, 3739-3752.

(28) Tai, W.; Chen, Z.; Barve, A.; Peng, Z.; Cheng, K. A novel rapamycin-polymer conjugate based on a new poly(ethylene glycol) multiblock copolymer. Pharm. Res. 2014, 31, 706-719.

(29) Simamora, P.; Alvarez, J. M.; Yalkowsky, S. H. Solubilization of rapamycin. Int. J. Pharm. 2001, 213, 25-29.

(30) Yáñez, J. A.; Forrest, M. L.; Ohgami, Y.; Kwon, G. S.; Davies, N. M. Pharmacometrics and delivery of novel nanoformulated PEG-bpoly $(\varepsilon$-caprolactone $)$ micelles of rapamycin. Cancer Chemother. Pharmacol. 2008, 61, 133-144.

(31) Karimi, M.; Bahrami, S.; Ravari, S. B.; Zangabad, P. S.; Mirshekari, H.; Bozorgomid, M.; Shahreza, S.; Sori, M.; Hamblin, M. R. Albumin nanostructures as advanced drug delivery systems. Expert Opin. Drug Delivery 2016, 13, 1609-1623.

(32) Bekdemir, A.; Stellacci, F. A centrifugation-based physicochemical characterization method for the interaction between proteins and nanoparticles. Nat. Commun. 2016, 7, No. 13121.

(33) Antognelli, C.; Trapani, E.; Delle Monache, S.; Perrelli, A.; Fornelli, C.; Retta, F.; Cassoni, P.; Talesa, V. N.; Retta, S. F. Data in support of sustained upregulation of adaptive redox homeostasis mechanisms caused by KRIT1 loss-of-function. Data in Brief 2018, 16, 929-938.

(34) Stern, S. T.; Adiseshaiah, P. P.; Crist, R. M. Autophagy and lysosomal dysfunction as emerging mechanisms of nanomaterial toxicity. Part. Fibre Toxicol. 2012, 9, No. 20.

(35) Mao, B.-H.; Tsai, J.-C.; Chen, C.-W.; Yan, S.-J.; Wang, Y.-J. Mechanisms of silver nanoparticle-induced toxicity and important role of autophagy. Nanotoxicology 2016, 10, 1021-1040.

(36) Ma, X.; Wu, Y.; Jin, S.; Tian, Y.; Zhang, X.; Zhao, Y.; Yu, L.; Liang, X.-J. Gold Nanoparticles Induce Autophagosome Accumulation through Size-Dependent Nanoparticle Uptake and Lysosome Impairment. ACS Nano 2011, 5, 8629-8639.

(37) Bibee, K. P.; Cheng, Y.-J.; Ching, J. K.; Marsh, J. N.; Li, A. J.; Keeling, R. M.; Connolly, A. M.; Golumbek, P. T.; Myerson, J. W.; Hu, G.; Chen, J.; Shannon, W. D.; Lanza, G. M.; Weihl, C. C.; Wickline, S. A. Rapamycin nanoparticles target defective autophagy in muscular dystrophy to enhance both strength and cardiac function. FASEB J. 2014, 28, 2047-2061.

(38) Choi, A. M. K.; Ryter, S. W.; Levine, B. Autophagy in Human Health and Disease. N. Engl. J. Med. 2013, 368, 1845-1846. 
(39) Corr, M.; Lerman, I.; Keubel, J. M.; Ronacher, L.; Misra, R.; Lund, F.; Sarelius, I. H.; Glading, A. J. Decreased KRIT1 expression leads to increased vascular permeability and modifies inflammatory responses in vivo. Arterioscler., Thromb., Vasc. Biol. 2012, 32, 27022710.

(40) Glading, A.; Han, J.; Stockton, R. A.; Ginsberg, M. H. KRIT-1/ CCM1 is a Rap1 effector that regulates endothelial cell-cell junctions. Int. J. Biochem. Cell Biol. 2007, 179, 247-254.

(41) Maddaluno, L.; Rudini, N.; Cuttano, R.; Bravi, L.; Giampietro, C.; Corada, M.; Ferrarini, L.; Orsenigo, F.; Papa, E.; Boulday, G.; Tournier-Lasserve, E.; Chapon, F.; Richichi, C.; Retta, S. F.; Lampugnani, M. G.; Dejana, E. EndMT contributes to the onset and progression of cerebral cavernous malformations. Nature 2013, 498, No. 492.

(42) Stockton, R. A.; Shenkar, R.; Awad, I. A.; Ginsberg, M. H. Cerebral cavernous malformations proteins inhibit Rho kinase to stabilize vascular integrity. J. Exp. Med. 2010, 207, 881-896.

(43) Zheng, X.; Xu, C.; Di Lorenzo, A.; Kleaveland, B.; Zou, Z.; Seiler, C.; Chen, M.; Cheng, L.; Xiao, J.; He, J.; Pack, M. A.; Sessa, W. C.; Kahn, M. L. CCM3 signaling through sterile 20-like kinases plays an essential role during zebrafish cardiovascular development and cerebral cavernous malformations. J. Clin. Invest. 2010, 120, 27952804.

(44) Guan, J.; Couldwell, W. T. Evaluating the Role of CCM1 Lossof-Function-Induced Endothelial-to-Mesenchymal Transition in Cavernous Malformation Development. World Neurosurg. 2013, 80, 444-446.

(45) Kovacic, J. C.; Mercader, N.; Torres, M.; Boehm, M.; Fuster, V. Epithelial- and Endothelial- to Mesenchymal Transition: from Cardiovascular Development to Disease. Circulation 2012, 125, 1795-1808.

(46) Borikova, A. L.; Dibble, C. F.; Sciaky, N.; Welch, C. M.; Abell, A. N.; Bencharit, S.; Johnson, G. L. Rho Kinase Inhibition Rescues the Endothelial Cell Cerebral Cavernous Malformation Phenotype. J. Biol. Chem. 2010, 285, 11760-11764.

(47) Panieri, E.; Santoro, M. M. ROS signaling and redox biology in endothelial cells. Cell. Mol. Life Sci. 2015, 72, 3281-3303.

(48) Sutradhar, K. B.; Amin, M. L. Nanotechnology in Cancer Drug Delivery and Selective Targeting. ISRN Nanotechnol. 2014, 2014, 112.

(49) Gadde, S. Multi-drug delivery nanocarriers for combination therapy. MedChem Comm 2015, 6, 1916-1929.

(50) Wu, J.; Ma, G.-H. Recent Studies of Pickering Emulsions: Particles Make the Difference. Small 2016, 12, 4633-4648.

(51) Laibinis, P. E.; Whitesides, G. M. omega.-Terminated alkanethiolate monolayers on surfaces of copper, silver, and gold have similar wettabilities. J. Am. Chem. Soc. 1992, 114, 1990-1995.

(52) Laibinis, P. E.; Whitesides, G. M. Self-assembled monolayers of $\mathrm{n}$-alkanethiolates on copper are barrier films that protect the metal against oxidation by air. J. Am. Chem. Soc. 1992, 114, 9022-9028.

(53) Ron, H.; Cohen, H.; Matlis, S.; Rappaport, M.; Rubinstein, I. Self-Assembled Monolayers on Oxidized Metals. 4. Superior nAlkanethiol Monolayers on Copper. J. Phys. Chem. B 1998, 102, 9861-9869.

(54) Caipa Campos, M. A.; Trilling, A. K.; Yang, M.; Giesbers, M.; Beekwilder, J.; Paulusse, J. M. J.; Zuilhof, H. Self-Assembled Functional Organic Monolayers on Oxide-Free Copper. Langmuir 2011, 27, 8126-8133.

(55) Laibinis, P. E.; Whitesides, G. M.; Allara, D. L.; Tao, Y. T.; Parikh, A. N.; Nuzzo, R. G. Comparison of the structures and wetting properties of self-assembled monolayers of $n$-alkanethiols on the coinage metal surfaces, copper, silver, and gold. J. Am. Chem. Soc. 1991, 113, 7152-7167.

(56) Shaheen, A.; Sturm, J. M.; Ricciardi, R.; Huskens, J.; Lee, C. J.; Bijkerk, F. Characterization of Self-Assembled Monolayers on a Ruthenium Surface. Langmuir 2017, 33, 6419-6426.

(57) Petrovykh, D. Y.; Kimura-Suda, H.; Opdahl, A.; Richter, L. J.; Tarlov, M. J.; Whitman, L. J. Alkanethiols on Platinum: Multi- component Self-Assembled Monolayers. Langmuir 2006, 22, 25782587.

(58) Li, Z.; Chang, S.-C.; Williams, R. S. Self-Assembly of Alkanethiol Molecules onto Platinum and Platinum Oxide Surfaces. Langmuir 2003, 19, 6744-6749.

(59) Fischer, M. J. Amine Coupling Through EDC/NHS: A Practical Approach. In Surface Plasmon Resonance; Mol, N., Fischer, M., Eds.; Humana Press, 2010; Vol. 627, pp 55-73.

(60) Brunetti, V.; Chibli, H.; Fiammengo, R.; Galeone, A.; Malvindi, M. A.; Vecchio, G.; Cingolani, R.; Nadeau, J. L.; Pompa, P. P. InP/ $\mathrm{ZnS}$ as a safer alternative to $\mathrm{CdSe} / \mathrm{ZnS}$ core/shell quantum dots: in vitro and in vivo toxicity assessment. Nanoscale 2013, 5, 307-317. 